\title{
A MULTIPLE EXCHANGE PROPERTY FOR BASES
}

\author{
CURTIS GREENE ${ }^{1}$
}

\begin{abstract}
Let $X$ and $Y$ be bases of a combinatorial geometry $G$, and let $A$ be any subset of $X$. Then there exists a subset $B$ of $Y$ with the property that $(X-A) \cup B$ and $(Y-B) \cup A$ are both bases of $G$.
\end{abstract}

I. Introduction. This paper is an outgrowth of a number of recent efforts to extend the methods of both linear algebra and classical invariant theory to the study of combinatorial geometries ([3], [4], [5], [6], [7]). One result of such efforts will, hopefully, be a completely satisfactory coordinatization theory for geometries-one which will include general techniques for automatically translating linear arguments into combinatorial ones. In spite of much encouraging work in this direction-and many interesting results - the full story apparently remains to be told.

As a result, the gap between "linear" and "nonlinear" combinatorial geometries sometimes seems embarrassingly large. There exist results which are easy to derive for linear geometries, using determinants or other techniques of linear algebra-but which are apparently much more difficult to prove by direct combinatorial methods.

This paper is devoted to the following example:

Theorem. Let $X$ and $Y$ be bases of a geometry $G$. Then for any subset $A \subseteq X$, there exists a subset $B \subseteq Y$ with the property that $(X-A) \cup B$ and $(Y-B) \cup A$ are both bases of $G$.

The case $|A|=1$ is a slight strengthening of the fact taken by Whitney [7] as the defining property for bases. It is easily proved by elementary arguments (see [2]).

If $G$ is linearly representable - that is, if the points of $G$ can be represented as points in a vector space $V$ over a field $F$ in such a way that dependence in $G$ corresponds to linear dependence in $V$-then the result

Received by the editors March 17, 1972.

AMS (MOS) subject classifications (1970). Primary 05B35; Secondary 15A03, $15 \mathrm{~A} 15$.

Key words and phrases. Combinatorial geometries, bases, exchange property, Laplace expansion.

${ }^{1}$ Supported in part by ONR N00014-67-A-0204-0063.

(c) American Mathematical Society 1973 
is an immediate consequence of the Laplace expansion theorem for determinants. The argument is as follows:

Suppose that $G$ has dimension $n$. We choose the elements of the basis $Y$ as coordinate vectors, and assume that the points of $G$ are represented accordingly as $n$-tuples over $F$. For any set $S \subseteq G$ of size $n$, we define $M(S)$ to be the $n \times n$ matrix whose columns are the vectors in $S$. Since $X$ is a basis, the matrix $M(X)$ is nonsingular. Applying the Laplace expansion theorem to the set $A$ of columns of $M(X)$, we obtain

$$
\operatorname{det} M(X)=\sum_{B \subseteq Y} \pm \operatorname{det} M((X-A) \cup B) \operatorname{det} M((Y-B) \cup A) \text {. }
$$

Since det $M(X) \neq 0$, some term on the right must be nonzero, and the result follows.

We remark that our combinatorial proof of this fact (given below) is not totally without interest in the linear case since it can easily be translated into an algorithm for actually finding the set $B$. Purely combinatorial versions of the exchange theorem can be obtained from the classical examples of combinatorial geometries-for example, if "bases" are replaced by spanning trees of a graph or maximal transversals of a family of sets. In these cases our proof provides a constructive method for carrying out the exchange.

2. Proof of the theorem. For the basic facts about combinatorial geometries, we refer the reader to [1] or [2].

We begin with a few elementary lemmas.

Lemma 1. Let $X$ and $Y$ be bases of $G$, and let $x \in X$. Let $d$ be the copoint spanned by $X-x$ and let $C$ be the unique circuit obtained by adding $x$ to $Y$. Then for any $y \in Y,(X-x) \cup y$ and $(Y-y) \cup x$ are both bases $\Leftrightarrow y$ and $y \in C$.

Proof. Immediate.

Lemma 2. Suppose $y_{1}, \cdots, y_{n-1}$ are independent and span a copoint $d_{0}$. Let $y_{1}^{\prime}, \cdots, y_{k}^{\prime}$ be points such that for each $i$,

(1) $y_{1}^{\prime} \vee y_{2}^{\prime} \vee \cdots y_{i}^{\prime} \vee y_{i+1} \vee \cdots \vee y_{n-1}$ is a copoint, say $d_{i}$.

(2) $d_{0} \neq d_{1} \neq \cdots \neq d_{k}$.

Then $\bigwedge_{j=0}^{k} d_{j}=y_{k+1} \vee \cdots \vee y_{n-1}$.

Proof. Immediate, by induction on $k$.

Lemma 3. Suppose $C_{1}, \cdots, C_{m}$ are circuits with the property that for each $i=2, \cdots, n$ there exists an element $y_{i} \in C_{i}-\bigcup_{j=1}^{i-1} C_{j}$. Then

$$
r\left(\bigcup_{1}^{m} C_{i}\right) \leqq\left|\bigcup_{1}^{m} C_{i}\right|-m \text {. }
$$


Proof. List the elements of $\bigcup_{1}^{m} C_{i}$ in order, beginning with $C_{1}, C_{2}$, etc. Then there are at least $m$ distinct elements which depend on their predecessors.

THEOREM. Let $X$ and $Y$ be bases of a geometry $G$. Then for any subset $A \subseteq X$, there exists a subset $B \subseteq Y$ with the property that $(X-A) \cup B$ and $(Y-B) \cup A$ are both bases of $G$.

Proof. Let $X=\left\{x_{1}, \cdots, x_{n}\right\}$ and $Y=\left\{y_{1}, \cdots, y_{n}\right\}$. We proceed by induction on the size of $A$. Assume that the theorem holds if $|A|=k$, and suppose now that $A=\left\{x_{1}, \cdots, x_{k+1}\right\}$. By assumption, we can exchange $x_{1}, \cdots, x_{k}$ for some subset of $Y$, which we denote by $y_{1}, \cdots, y_{k}$. Thus

$$
\begin{aligned}
X^{\prime} & =y_{1}, \cdots, y_{k}, x_{k+1}, \cdots, x_{n} \text { and } \\
Y^{\prime} & =x_{1}, \cdots, x_{k}, y_{k+1}, \cdots, y_{n}
\end{aligned}
$$

are both bases. The idea of the proof is as follows: we attempt to exchange $x_{k+1}$ for one of the $y$ 's in $Y^{\prime}$. If this is impossible, we exchange certain $y^{\prime}$ s in $X^{\prime}$ for $y$ 's in $Y^{\prime}$ until it becomes possible. The proof consists of showing that an appropriate sequence of switches can always be found.

Technically, it turns out that we cannot always switch $y$ 's in such a way that both sets remain bases. In our proof, we require only that the set $X^{\prime}-x_{k+1}$ have rank $n-1$ at each step. We use the following notation:

$$
\begin{aligned}
X^{\prime} & =U_{X} \cup U_{Y}, \quad U_{X}=\left\{x_{k+1}, \cdots, x_{n}\right\}, \quad U_{Y}=\left\{y_{1}, \cdots, y_{k}\right\}, \\
Y^{\prime} & =V_{X} \cup V_{Y}, \quad V_{X}=\left\{x_{1}, \cdots, x_{k}\right\}, \quad V_{Y}=\left\{y_{k+1}, \cdots, y_{n}\right\}, \\
d_{0} & \left.=\vee\left(X^{\prime}-x_{k+1}\right) \quad \text { (the copoint obtained by removing } x_{k+1} \text { from } X^{\prime}\right), \\
C_{0} & \left.=c\left(x_{k+1}, Y^{\prime}\right) \quad \text { (the circuit obtained by adding } x_{k+1} \text { to } Y^{\prime}\right) \\
& =x_{k+1} \cup C_{X}^{0} \cup C_{Y}^{0}, \quad C_{X}^{0} \subseteq X, \quad C_{Y}^{0} \subseteq Y, \\
C_{i} & =c\left(y_{i}, Y^{\prime}\right) \quad\left(\text { defined for } y_{i} \in U_{Y}\right) \\
& =y_{i} \cup C_{X}^{i} \cup C_{Y}^{i}, \quad C_{X}^{i} \subseteq X, \quad C_{Y}^{i} \subseteq Y .
\end{aligned}
$$

If there exists a $y \in C_{y}^{0}$ with $y \leq d_{0}$, then we can stop immediately for, by Lemma $1, X^{\prime}-x_{k+1} \cup y$ and $Y^{\prime}-y \cup x_{k+1}$ are both bases. So from now on we assume that $y \leqq d_{0}$ for all $y \in C_{y}^{0}$.

We define an admissible sequence of exchanges ("admissible sequence" for short) to be a sequence of pairs

$$
\left(y_{1}, y_{1}^{\prime}\right), \cdots,\left(y_{p}, y_{p}^{\prime}\right)
$$


satisfying the following conditions:

(i) $y_{i} \in U_{Y}, y_{1}^{\prime} \in V_{Y}-C_{0}, i=1, \cdots, p$.

(ii) For $i=1, \cdots, p, \bigvee\left(X^{\prime}-x_{k+1}-y_{1} \cdots-y_{i} \cup y_{i}^{\prime} \cup \cdots \cup y_{i}^{\prime}\right)$ is a copoint, hereafter denoted by $d_{12 \cdots i}$.

(iii) For $i=1, \cdots, p, Y^{\prime}-y_{1}^{\prime} \cdots-y_{i}^{\prime} \cup y_{1} \cup \cdots \cup y_{i}$ is a basis, hereafter denoted by $Y_{12 \ldots i}^{\prime}$.

(iv) $d_{0} \neq d_{1} \neq d_{12} \neq \cdots \neq d_{12 \cdots p}$.

Thus admissible sequences are sequences of exchanges of elements in $U_{Y}$ for elements in $V_{Y}-C_{0}$ which preserve a copoint-basis pair and have the property that each new copoint so obtained is distinct from the previous one. Condition (iv) is equivalent to requiring that $y_{1}^{\prime} d_{0}$ and $y_{i+1}^{\prime} d_{1 \cdots i}$ for $i=1, \cdots, p-1$.

We define

$Q=\left\{d \mid d=d_{1 \ldots p}\right.$ for some admissible sequence $\} \cup\left\{d_{0}\right\}$,

$S=\left\{y_{i} \in U_{\boldsymbol{Y}} \mid\right.$ there exists an admissible sequence ending in $\left.\left(y_{i}, y_{i}^{\prime}\right)\right\}$, and

$$
T=U_{Y}-S \text {. }
$$

Thus $T$ is the set of elements in $U_{Y}$ which are never switched in any admissible sequence. It may of course be empty.

The rest of the proof consists of showing that there exists some admissible sequence which leads to a situation in which $x_{k+1}$ can be exchanged. More precisely, we show that for some sequence there exists $y \in C_{Y}^{0}$ with the property that

$$
X^{\prime}-x_{k+1}-y_{1}-\cdots-y_{p} \cup y_{1}^{\prime} \cdots y_{p}^{\prime} \cup y
$$

and

$$
Y_{12 \cdots p}^{\prime}-y \cup x_{k+1}
$$

are both bases. To verify this, assume the contrary - that is, no admissible sequence leads to the situation just described. We complete the proof with a series of seven observations, leading to a contradiction:

(1) $y \leqq d$ for all $y \in C_{Y}^{0}$ and all $d \in Q$.

Proof. We have already shown that $y \leqq d_{0}$ for all $y \in C_{Y}^{0}$. Suppose now that $d=d_{12 \cdots p}$. Then $C_{0}=c\left(x_{k+1}, Y^{\prime}\right)=c\left(x_{k+1}, Y_{12 \ldots p}^{\prime}\right)$, since condition (i) guarantees that no $y_{i}^{\prime}$ removed from $Y^{\prime}$ is in $C_{0}$. This means that the elements exchangeable for $x_{k+1}$ in $Y^{\prime}$ and $Y_{12 \ldots p}^{\prime}$ are identical. If there exists $y \in C_{Y}^{0}$ with $y \leq d_{12 \cdots p}$, then $X^{\prime}-x_{k+1}-y_{1}-\cdots-y_{p} \cup y_{1}^{\prime} \cup \cdots \cup$ $y_{p}^{\prime} \cup y$ and $Y_{12 \ldots p}^{\prime}-y \cup x_{k+1}$ are both bases. Since this was assumed not to occur, the conclusion follows.

(2) Let $y_{j} \in T$. Then $y \leqq d$ for all $y \in C_{Y}^{j}$ and all $d \in Q$. 
Proof. It is clear that $y \leqq d_{0}$ for all $y \in C_{Y}^{j}$, since otherwise $\left(y_{j}, y\right)$ is an admissible sequence of length one. (Note that $y \leq d_{0}$ implies $y \notin C_{0}$, so that (i) is satisfied.) If $d=d_{12 \cdots p}$, we may suppose that $y \leqq d^{\prime}$ for all $y \in C_{Y}^{j}$ and $d^{\prime}=d_{0}, d_{1}, \cdots, d_{12 \cdots(p-1)}$. As before, $C_{j}=c\left(y_{j}, Y^{\prime}\right)=$ $c\left(y_{j}, Y_{12 \ldots p}^{\prime}\right)$, since $y_{1}^{\prime} d_{0}$ and $y_{i+1}^{\prime} d_{12 \cdots i}$ for $i=1, \cdots, p-1$, and so every $y_{i}^{\prime}$ removed is outside of $C_{j}$ (by the inductive hypothesis). If there exists $y \in C_{T}^{j}$ with $y \leq d_{12 \cdots p}$, then $\left(y_{1}, y_{1}^{\prime}\right), \cdots,\left(y_{p}, y_{p}^{\prime}\right),\left(y_{j}, y\right)$ is an admissible sequence, contradicting the assumption that $y_{j} \in T$. (Again, $y \notin C_{0}$ since $y \leqq d_{12 \cdots p}$ for all $y \in C_{Y}^{0}$, by (1).) This completes the proof.

(3) Let $\beta=\bigwedge_{d \in Q} d$. Then $y \leqq \beta$ for every $y \in Y$ which appears among the circuits $C_{0}$ and $C_{i}, y_{i} \in T$.

Proof. This is an immediate consequence of (1) and (2), and the definition of $T$.

We pause briefly at this point to sketch the idea behind the rest of the proof. We will show that (3) is impossible because too many $x$ 's "depend" on the $y$ 's in $\beta$. In fact, the remaining steps show that adding certain $x$ 's to $\beta$ results in a flat of dimension $<n$ which spans all the $x$ 's, contradicting the fact that $X$ is a basis.

(4) $\beta=\bigvee\left(X^{\prime}-x_{k+1}-S\right)$.

Proof. By Lemma 2, $d_{0} \wedge d_{1} \wedge \cdots \wedge d_{12 \cdots p}=\bigvee\left(X^{\prime}-x_{k+1}-y_{1}-\cdots-y_{p}\right)$ for any admissible sequence $\left(y_{1}, y_{1}^{\prime}\right), \cdots,\left(y_{p}, y_{p}^{\prime}\right)$. The result follows immediately from this.

(5) Let

$$
\begin{gathered}
C_{X}=\left(\bigcup_{y_{j} \in T} C_{X}^{j}\right) \cup C_{X}^{0}, \quad C_{Y}=\left(\bigcup_{y_{j} \in T} C_{Y}^{j}\right) \cup C_{Y}^{0} \cup T, \\
R_{X}=V_{X}-C_{X} \quad \text { and } \quad \alpha=\vee C_{X} \vee \vee C_{Y} \vee \vee R_{X} .
\end{gathered}
$$

Then (i) $r(\alpha) \leqq\left|C_{X}\right|+\left|C_{Y}\right|+\left|R_{X}\right|-|T|-1=\left|C_{Y}\right|+k-|T|$ and $r(\alpha \wedge \beta) \geqq\left|C_{Y}\right|$.

Proof. To verify (i), we observe that the set $S=C_{0} \cup \cup_{Y_{i} \in T} C_{j}$ has rank $\leqq|S|-|T|-1$, by Lemma 3 . Since $\alpha$ is obtained by adding the set $R_{X}$ to $S$, the inequality follows. Inequality (ii) follows immediately from (3) and the fact that $C_{Y}$ is independent.

(6) $r(\alpha \vee \beta) \leqq n-1$.

Proof. By the submodular inequality, $r(\alpha \vee \beta) \leqq r(\alpha)+r(\beta)-r(\alpha \wedge \beta)$. But $r(\beta)=n-k-1+|T|$, and substituting the results of (5) gives the inequality $r(\alpha \vee \beta) \leqq\left(\left|C_{Y}\right|+k-|T|\right)+(n-k-1+|T|)-\left|C_{Y}\right|=n-1$.

(7) This is impossible. 
Proof. $\alpha$ contains $x_{k+1}$, since it contains $C_{0}$, and also every $x \in V_{X}$. $\beta$ contains every $x \in U_{X}$ except $x_{k+1}$, by (4). Hence $\alpha \vee \beta$ contains every $x \in X$, and so must have rank $n$.

This contradiction shows that some admissible sequence must lead to a "switchable" $y$, and the proof is complete.

\section{REFERENCES}

1. H. Crapo and G.-C. Rota, On the foundations of combinatorial theory. II. Combinatorial geometries, Studies in Appl. Math. 49 (1970), 109-133. MR 44 \#3882.

2. C. Greene, Lectures on combinatorial geometries, Bowdoin College, Brunswick, Me., 1971 (mimeographed notes).

3. C. Greene, G.-C. Rota and N. White, Coordinates and combinatorial geometries (to appear).

4. G.-C. Rota, Combinatorial theory, old and new, Proc. Internat. Congress Math. (Nice, 1970), vol. 3, Gauthier-Villars, Paris, 1971, pp. 229-234.

5. —_ Combinatorial theory, Bowdoin College, Brunswick, Me., 1971. (mimeographed notes).

6. N. White, Brackets and combinatorial geometries, Thesis, Harvard University, Cambridge, Mass., 1971.

7. W. Whitely, Logic and invariant theory, Thesis, M.I.T., Cambridge, Mass., 1971.

8. H. Whitney, On the abstract properties of linear dependence, Amer. J. Math. 57 (1935), 509.

Department of Mathematics, Massachusetts Institute of Technology, CamBRIDGE, MASSACHUSETTS 02139 